\title{
Larissa Zakharova, De Moscou aux terres les plus lointaines. Communications, politique et société en URSS
}

Paris, Éditions de l'EHESS, 2020

\section{Valérie Schafer}

\section{OpenEdition}

\section{Journals}

Édition électronique

URL : https://journals.openedition.org/artefact/10658

DOI : 10.4000/artefact.10658

ISSN : 2606-9245

\section{Éditeur :}

Association Artefact. Techniques histoire et sciences humaines, Presses universitaires du Midi

\section{Édition imprimée}

Date de publication : 7 octobre 2021

Pagination : 495-500

ISBN : 978-2-8107-0755-3

ISSN : 2273-0753

Référence électronique

Valérie Schafer, «Larissa Zakharova, De Moscou aux terres les plus lointaines. Communications, politique et société en URSS », Artefact [En ligne], 14 | 2021, mis en ligne le 26 août 2021, consulté le 03 septembre 2021. URL : http://journals.openedition.org/artefact/10658 ; DOI : https://doi.org/10.4000/ artefact. 10658

Artefact, Techniques, histoire et sciences humaines est mise à disposition selon les termes de la Licence Creative Commons Attribution - Pas d'Utilisation Commerciale - Pas de Modification 4.0 International. 
Larissa Zakharova, De Moscou aux terres les plus lointaines. Communications, politique et société en URSS

Paris, Éditions de l'EHESS, 2020, 333 pages

$\mathrm{E}$

n 2016, Benjamin Peters offrait une plongée dans les stratégies de mise en place des réseaux informatiques dans le monde soviétique, de la fin des années 1950 à la fin des années 1980, et notamment dans le projet All-State Automated System (OGAS). Le titre de son ouvrage How Not to Network a Nation montrait les difficultés à penser un système de réseaux à la fois décentralisé localement, mais centralisé autour de Moscou ${ }^{1}$. Son analyse permettait d'une part de sortir des binarismes opposant monde soviétique et états-unien, et d'autre part de proposer une vision techno-politique plus complexe qu'il n'y paraît au premier abord. Le livre de Larissa Zakharova rejoint cette seconde ambition, tout en livrant une approche différente de cette histoire, qui montre que les réseaux n'ont bien sûr pas attendu l'informatique. C'est notamment en partant des réseaux postaux, télégraphiques et téléphoniques qu'elle analyse les enjeux, réalités et limites de la mise en réseau en URSS au cours du Xxe siècle. Le livre $D e$ Moscou aux terres les plus lointaines permet ainsi d'explorer des aspects de l'histoire des communications dans le monde soviétique à contre-courant pourrait-on dire du titre How Not to Network a Nation : il rend palpable dans toutes ses nuances, subtilités, complexités, un monde d'échanges et de communications, qui (s')adapte, bouge, contourne, et ce au moyen d'une écriture élégante et d'un matériel archivistique riche et finement exploité.

Sans aucun doute cet ouvrage est important non seulement pour les études soviétiques mais aussi pour l'histoire des communications. L'introduction de l'ouvrage le démontre, en présentant les nombreux thèmes qui sont explorés avec attention : que ce soit la notion de contrôle et de modernité, ou la question des mobilités et de l'espace, celle des temporalités politiques et techniques (qui s'entremêlent étroitement), mais aussi les développements dédiés aux infrastructures ou aux transferts techniques.

On notera d'emblée deux apports majeurs de l'ouvrage qui suffiraient à eux seuls à démontrer l'ampleur du travail de recherche accompli :

1. Peters, 2016. 
- le cadre chronologique large d'une part, qui impliquait de penser des évolutions et de ne pas présenter un tableau figé. Le pari est réussi, par exemple quand l'auteure montre à propos des restrictions sur le télégraphe que la hiérarchie des communications est sans cesse repensée (p. 41), ou quand elle présente les continuités, par exemple dans les pratiques matérielles du secret (p. 144 et ss.), mais aussi les reconfigurations communautaires et celles des communications (p. 189 et ss) ;

- le choix de mêler postes, télégraphe et télécommunications d'autre part. S'il peut paraître assez logique dans une perspective communicationnelle, rares sont les historiens à l'instar de Larissa Zakharova ou de Léonard Laborie à avoir fait tenir ces différentes infrastructures de concert.

Ajoutons qu'en se plaçant notamment dans le troisième chapitre au plus près des utilisateurs, saisis au prisme de leurs échanges épistolaires, Larissa Zakharova apporte aussi une dimension multi-scalaire et une approche de cette histoire à la fois par le haut (vision politique, ingénieuriale) et par le bas (correspondances, études de parcours communicationnels individuels). Comme le notait Laurent Thévenot : "Les objets de recherche sur lesquels Larissa Zakharova a travaillé ont souvent été des objets matériels, ce qui inscrit sa recherche historique et sociologique dans l'extension nouvelle résultant de l'attention portée aux choses et aux techniques. Cependant, sa démarche élargit l'approche de l'environnement matériel en le montrant pris tout autant dans des actions et des politiques de dimension publique que dans l'intimité personnelle des usagers ${ }^{2}$. "

Croisant ainsi le poids des structures, institutions, censures, régulations et celui des communautés en une grille évolutive dans le temps, de Lénine à Brejnev (p. 22-23), Larissa Zakharova explore les liens forts et faibles qui s'expriment dans ces régimes communicationnels, et ce en une approche à la fois politique, technique et sociétale. Elle prolonge ainsi utilement une approche qu'elle avait entamée dans l'article "Concevoir l'efficacité des communications en Union soviétique, fin des années 1920-début des années $1930^{3}$ ", mais qu'elle enrichit aussi indéniablement en termes d'acteurs (on rencontre au fil du livre aussi bien des radioamateurs à la page 106, sujets aux sarcasmes des ingénieurs, que des dactylographes,

2. Thévenot, 2019, p. 595.

3. Zakharova, 2015. 
chers à Delphine Gardey, p. 130-131), de stratégies, d'usages et de controverses. Elle interrogeait déjà dans cet article la quête de rationalité dans le secteur des communications et la recherche de rentabilité, en s'appuyant sur les perspectives des économistes et des ingénieurs. Si cette question n'était pas dénuée de controverses, celles que Larissa Zakharova nous décrit dans De Moscou aux terres les plus lointaines sont nombreuses, reconstruites de manière passionnante et avec la même minutie, à l'instar de celle qui touche à l'automatisation (p. 87 et ss.). On y trouve de grands acteurs industriels européens tels Ericsson et Siemens, mais aussi toutes les divergences d'appréciation et d'intérêt entre acteurs (p. 95 et ss.) qui dévoilent des conflits hiérarchiques, institutionnelles, des logiques et visions de la modernité concurrentes. On y retrouve également toute la finesse de ses réflexions précédentes sur les paradoxes de la "modernité soviétique ", ou pour suivre Cyril Lemieux ses contradictions ${ }^{4}$ :

En tenant fermement cette position méthodologique internaliste, Larissa Zakharova manifestait une fois encore une attitude sociologique. Celleci lui permettait de voir la modernité soviétique 'de l'intérieur', sans jamais en subordonner l'analyse à des références et à des processus exogènes, mais néanmoins en prenant en compte ces derniers, tels qu'appréhendés par les acteurs depuis cet intérieur même. Plus encore, cette attitude tendait à la conduire vers ce que l'on serait tenté d'appeler une 'critique interne' de l'expérience soviétique. Par opposition à la critique externe qui rapporte cette expérience, pour la juger, aux canons de la modernité euroaméricaine, une telle critique interne repose en premier lieu sur la prise au sérieux de l'utopie de modernisation développée par le régime soviétique et, en second lieu, sur l'analyse des contradictions internes à la société soviétique, contradictions à travers lesquelles se manifeste l'extrême difficulté du régime à faire advenir l'utopie dans l'effectivité des pratiques sociales

Historique dans son approche, ce livre n'en a pas moins utilement recours à la sociologie, et l'on retrouve ici les croisements interdisciplinaires qu'avait déjà menés avec succès l'auteure dans S'habiller à la soviétique. La mode et le dégel en URSS ${ }^{5}$, pour enrichir une réflexion qui bénéficie sur la com-

4. Lemieux, 2019, p. 588.

5. Zakharova, 2011. 
munication interpersonnelle d'analyses sur la confiance, la subjectivation, la solidarité ou encore la conscience et la réflexivité (p. 16). Ce n'est pas un des moindres défis relevés par cet ouvrage que de croiser des sources officielles, les fonds de l'état, dans la première partie, et des documents privés, notamment familiaux (voir la section Sources, p. 315 et ss). On retrouve ici l'intérêt de l'auteure pour des archives personnelles, des traces individuelles privées, qu'elle avait eu plaisir à exploiter dès le début de ses recherches, notamment sur l'habitat soviétique et la redistribution du logement et qu'elle confiait à Alain Blum en 2019 : " Je trouvais intéressant de raconter les histoires de certains habitants et pour ce projetlà, je suis allée aux archives de SaintPétersbourg et je suis tombée sur un fonds qui expliquait la répartition, la redistribution des logements et des biens après la révolution dans cet immeublelà. C'était un fonds de l'économie municipale et ils avaient des dossiers de ce type pour plusieurs adresses dans la ville ${ }^{6}$. "

L'approche multi-scalaire mais aussi au plus près des habitats et de ses habitants est sensible quand Larissa Zakharova évoque par exemple le manque de coopération entre spécialistes en téléphonie et experts en urbanisme (p. 180-181), fournissant en outre d'intéressantes données chiffrées sur la présence d'appareils téléphoniques dans les immeubles. On ne peut que de découvrir avec intérêt aussi, en suivant avec elle ces installations et les occupants des immeubles, une mention du télétravail (p. 182), quand un membre correspondant de l'académie des sciences de l'Union Soviétique et membre titulaire de l'académie des sciences d'Ukraine sollicite une liaison téléphonique par fils aériens pour travailler et suivre ses doctorants de son domicile en raison de problèmes de santé (p. 182). Ce n'est qu'un exemple des multiples anecdotes qui émaillent un récit vivant et rendent palpables des pratiques et procédés que l'auteure analyse aussi en terme de justice sociale, égalitaire ou distributive, suivant en cela Nancy Fraser (p. 184). Ces récits donnent chair à une analyse qui ne fait jamais l'impasse sur les structures macro mais aussi, par des procédés de micro-histoire, les met en relation avec l'histoire des subjectivités et l'histoire sociale, comme Larissa Zakharova l'évoquait déjà dans " Des engagements d'une écrivaine en URSS. Le cas de Natal'ja Četunova », notant : "L'histoire des subjectivités et l'histoire sociale des lettres destinées à la presse et aux autorités 
étudient les écrits en tant qu'ils sont des rapports de communication essentiellement verticaux. Cependant, pour comprendre les principes du "vivre ensemble" et la multitude des engagements dans la société soviétique, il est nécessaire de saisir les personnes dans la complexité de leurs liens "verticaux" et "horizontaux"”."

On croisera ainsi un pianiste surveillé par le KGB (p. 230), ou ce grand épistolier qu'est Šejgal (p. 277 et ss.), qui notamment en avril 1943 rédige plus de cinquante lettres en quatre jours à ses proches, une correspondance exploitée avec minutie par l'auteure pour celles des lettres qui existent toujours.

On aurait envie à l'issue de ce travail impressionnant d'engager le dialogue avec Larissa Zakharova sur l'iconographie de ces communications et de cette modernité, de revenir avec elle sur les asymétries de pouvoir et de communication ou les rapports de genre qui traversent l'ouvrage, sur les héritages actuels de cette histoire. Mais notait Sophie Cœuré : "Le dialogue est ici interrompu : Larissa Zakharova est décédée d'un cancer en mars 2019, quelques semaines après m'avoir envoyé la conclusion de son travail [...]. Ainsi, elle a mené presque à son terme son projet d'accomplissement scientifique, sans les dernières corrections que son perfectionnisme aurait souhaitées, sans non plus la brisure nette des travaux académiques interrompus par les tragédies de l'histoire et dont le destin posthume prix, soutenances, publications - reste à étudier ${ }^{8}$ ". Cette édition, établie par Grégory Dufaud, par son apport majeur à l'historiographie des études soviétiques comme de l'histoire des communications et des techniques, s'impose comme une recherche incontournable. Son destin posthume sera indéniablement assuré par sa qualité, et avec lui celui d'une chercheuse irremplaçable par sa capacité à nous entraîner en des terres lointaines avec rigueur, originalité et sensibilité.

Valérie SCHAFER

7. Zakharova, 2019, p. 598.

8. Cœuré, 2019, p. 568. 


\section{Sources et Bibliographie}

Blum Alain, Zakharova Larissa, " "Comment je me suis retrouvée à étudier l'histoire soviétique...”. Entretien avec Larissa Zakharova, recueilli par Alain Blum à Moscou le 25 janvier 2019 ", Cahiers du monde russe, 2019/2-3, vol. 60, p. 553-566, https://www.cairn.info/revue-cahiers-du-monde-russe-2019-2page-553.htm.

Ceeuré Sophie, "Communications, pouvoir et société en URSS de Larissa Zakharova (1977-2019). Rapport sur le manuscrit inédit d'habilitation à diriger des recherches, in abstentia. Une œuvre posthume ", Cahiers du monde russe, 2019/2-3, vol. 60, p. 567-576, https://www.cairn.info/revue-cahiers-dumonde-russe-2019-2-page-567.htm.

Lemieux Cyril, "Une historienne profondément sociologue ", Cahiers $d u$ monde russe, 2019/2-3, vol. 60, p. 585-592, https://www.cairn.info/revue-cahiers-dumonde-russe-2019-2-page-585.htm.

Peters Benjamin, How Not to Network a Nation. The Uneasy History of the Soviet Internet, The MIT Press, Cambridge MA, 2016.

ThÉvenot Laurent, "Concevoir la communication soviétique. Le discernement compréhensif de Larissa Zakharova ", Cahiers du monde russe, 2019/2-3, vol. 60, p. 593-596, https://www.cairn.info/revue-cahiers-du-monde-russe2019-2-page-593.htm.

ZaKharova Larissa, S'habiller à la soviétique. La mode et le dégel en URSS, CNRS Éditions, Paris, 2011.

ZaKharova Larissa, "Concevoir l'efficacité des communications en Union soviétique, fin des années 1920-début des années 1930 ", Histoire \& mesure, 1, 2015, https://journals.openedition.org/histoiremesure/5178.

Zakharova Larissa, "Des engagements d'une écrivaine en URSS. Le cas de Natal'ja Četunova ", Cahiers du monde russe, 2019/2-3, vol. 60, p. 597-618, https://www.cairn.info/revue-cahiers-du-monde-russe-2019-2-page-597.htm. 